\title{
Effect of paricalcitol and GcMAF on angiogenesis and human peripheral blood mononuclear cell proliferation and signaling
}

\author{
Stefania Pacini ${ }^{1}$, Gabriele Morucci ${ }^{1}$, Tiziana Punzi ${ }^{1}$, \\ Massimo Gulisano 1, Marco Ruggiero ${ }^{2}$, \\ Marcello Amato ${ }^{3}$, Stefano Aterini ${ }^{3}$
}

\author{
${ }^{1}$ Department of Anatomy, Histology and Forensic Medicine, \\ University of Firenze, Firenze - Italy \\ ${ }^{2}$ Department of Experimental Pathology and Oncology, \\ University of Firenze, Firenze - Italy \\ ${ }^{3}$ Department of Nephrourology, Misericordia e Dolce Hospital, \\ Prato - Italy
}

\section{Abstract}

Background: In addition to its role in calcium homeostasis and bone mineralization, vitamin $D$ is involved in immune defence, cardiovascular function, inflammation and angiogenesis, and these pleiotropic effects are of interested in the treatment of chronic kidney disease. Here we investigated the effects of paricalcitol, a nonhypercalcemic vitamin D analogue, on human peripheral blood mononuclear cell proliferation and signaling, and on angiogenesis. These effects were compared with those of a known inhibitor of angiogenesis pertaining to the vitamin $D$ axis, the vitamin D-binding protein-derived Gc-macrophage activating factor (GcMAF).

Methods: Since the effects of vitamin $\mathrm{D}$ receptor agonists are associated with polymorphisms of the gene coding for the receptor, we measured the effects of both compounds on mononuclear cells harvested from subjects harboring different Bsml polymorphisms.

Results: Paricalcitol inhibited mononuclear cell viability with the bb genotype showing the highest effect. GcMAF, on the contrary, stimulated cell proliferation, with the bb genotype showing the highest stimulatory effect. Both compounds stimulated 3'-5'-cyclic adenosine monophosphate formation in mononuclear cells with the highest effect on the bb genotype. Paricalcitol and GcMAF inhibited the angiogenesis induced by proinflammatory prostaglandin E1.

Conclusions: Polymorphisms of the vitamin D receptor gene, known to be associated with the highest re- sponses to vitamin $D$ receptor agonists, are also associated with the highest responses to GcMAF. These results highlight the role of the vitamin $D$ axis in chronic kidney disease, an axis which includes vitamin $D$, its receptor and vitamin D-binding protein-derived GcMAF.

Key words: Angiogenesis, Mononuclear cells, Paricalcitol, Proliferation

\section{INTRODUCTION}

The vitamin $D$ axis includes vitamin $D$, the polymorphic vitamin D receptor (VDR) and the vitamin D-binding protein (Gc-globulin) (1), which is the precursor of the potent Gcmacrophage activating factor (GcMAF) showing pleiotropic effects on the immune system and angiogenesis (2). The interest in the role of the vitamin D axis in chronic kidney disease (CKD) is demonstrated by the rising number of publications on these compounds. Thus, a search of www. PubMed.org (accessed on April 14, 2011) for "vitamin D and chronic kidney disease" yielded 2,658 papers; "vitamin D receptor and chronic kidney disease," 266 papers; "vitamin D-binding protein and chronic kidney disease," 19 papers. According to a recent review, the role of GcMAF in CKD has been hypothesized, but it has still to be studied (3).

It is well established that vitamin $\mathrm{D}$ and the nonhypercalcemic VDR agonists (analogues) exert their effects through the interaction with VDR, a protein coded for by a gene har- 
boring polymorphisms associated with bone turnover and density (4), diabetes, cancer, cardiovascular disease, as well as with chronic inflammation (5). VDR polymorphisms also influence the immune system response to vitamin $D$, and cells carrying certain alleles are less responsive to vitamin D $(6,7)$. As recently hypothesized (3), in addition to vitamin $D$, another component of the axis, GcMAF, could be useful in controlling those harmful effects of inflammation and excessive immune system stimulation commonly observed in CKD (8) thanks to its pleiotropic effects. Thus, the components of the vitamin $D$ axis are also involved in the control of angiogenesis, a process frequently deregulated in CKD (9), and vitamin D and GcMAF show antiangiogenic properties that could be useful in controlling some of the effects of excessive inflammation (2, 10-14).

Considering the multifaceted role of the components of the vitamin $\mathrm{D}$ axis in $\mathrm{CKD}$, in this study first we investigated the effects of 2 components of the axis - a nonhypercalcemic vitamin D analogue, paricalcitol, and GCMAF - on the angiogenesis stimulated by proinflammatory prostaglandin E1 (PGE1) in the chick embryo chorioallantoic membrane (CAM) assay. Then, considering the role of peripheral blood mononuclear cells (PBMCs) in initiating and maintaining a proinflammatory state in CKD (15), we evaluated the effects of the 2 compounds on human PBMCs. Since the effects of vitamin $D$ and its analogues depend upon VDR polymorphisms, we evaluated the association between Bsml polymorphisms of the VDR gene and the response of human PBMCs to paricalcitol and GcMAF. Finally, since the relationship between the cAMP signal transduction pathway induced by vitamin $D$ and GcMAF and VDR polymorphisms has received little attention so far, and to establish the role of VDR genotypes in modulating cAMP formation, we investigated cAMP formation in human PBMCs from donors with different VDR polymorphisms.

\section{SUBJECTS AND METHODS}

PBMCs were collected from healthy volunteers $(n=68)$ in accord with the Declaration of Helsinki; the donors gave their informed consent. Permission to perform this study was granted by the local ethics committees (Azienda Ospedaliera Universitaria Careggi and Prato Hospital). Prof. M. Gulisano supervised compliance with local ethics committee guidelines.

Whole venous blood $(5 \mathrm{~mL})$ was treated with ethylenediaminetetraacetic acid (EDTA) as anticoagulant. The separation procedure to obtain PBMCs was performed with Polymorphoprep (Axis-Shield PoCAS, Oslo, Norway) following the manufacturer's instructions. PBMCs were cultured as described (11). Treatments with paricalcitol (10-100 nM) or with
GcMAF $(0.01-1 \mathrm{ng} / \mathrm{mL})$ were performed in complete culture medium (2,12-14). Each condition was replicated in quadruplicate samples, and each subject served as internal control. Each experiment was replicated 3 times. With regard to VDR genotypes (e.g., the homozygous bb genotype), we refer to the results obtained from subjects harboring the indicated genotype. GcMAF was obtained from www.gcmaf.eu $(2,11)$. Assessment of cell viability was determined by Calbiochem Rapid Cell Proliferation Kit (Calbiochem D.B.A., Milan, Italy); cAMP levels were measured by a competitive EIA assay (Cyclic AMP EIA Kit; Cayman Chemical, Ann Arbor, MI, USA). In both cases, differences between experimental points were evaluated by Student's $t$-test.

The VDR restriction site polymorphism was evaluated on genomic DNA extracted from peripheral blood cells using a QIAamp DNA Blood Mini Kit (QIAGEN S.p.a., Milan, Italy) and amplified by polymerase chain reaction. Amplification and digestion for the detection of the Bsml polymorphism were performed as described previously (16). Absence or presence of the Bsml restriction site was denominated "B" and "b," respectively.

CAM assay was performed as described previously (17). Gelatin sponges containing $5 \mu \mathrm{L}$ phosphate-buffered saline (PBS) or PGE1 were used respectively as negative and positive controls. In each experiment we used 6 eggs per experimental point, and each experiment was repeated 3 times. Eggs were examined by stereomicroscopy, and positive angiogenesis was considered to have been found when new microvessels had developed around the sponge. Images of the CAM were captured using a digital camera connected to the microscope. Differences between experimental points were evaluated by Student's $t$-test.

\section{Results}

\section{Effects of paricalcitol and GcMAF on angiogenesis}

To evaluate the effects of the nonhypercalcemic vitamin $D$ analogue paricalcitol and those of a known inhibitor of angiogenesis (GcMAF) on angiogenic signaling pathways, we tested their effects in basal conditions and when administered together with a powerful stimulator of angiogenesis: PGE1 (1 mg/mL). Paricalcitol or GcMAF did not alter basal angiogenesis (Fig. 1) or chick embryo development (not shown). As expected, PGE1 (1 mg/mL) strongly stimulated angiogenesis, as previously described (18); blood vessels with an irregular course and frequent branching ("spokedwheel" pattern) surrounded the gelatin sponges. Numerous tortuous vessels infiltrated the sponges and often modified 


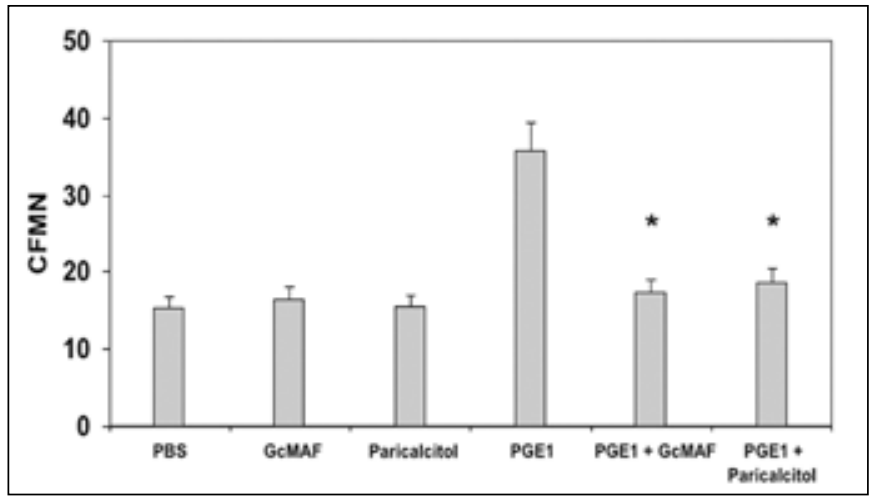

Fig. 1 - Quantitative evaluation of angiogenesis on chorioallantoic membrane assay. The average number of blood vessels, expressed as circumfocal microvessel number (CFMN), derived from scoring small $(<1 \mathrm{~mm}$ diameter) and tortuous $\mathrm{mi}-$ crovessels, for each of the experimental conditions. The different compounds were used at the following concentrations: Gc-macrophage activating factor (GcMAF) $1 \mathrm{ng} / \mathrm{mL}$, paricalcitol $100 \mathrm{nM}$ and prostaglandin E1 (PGE1) $1 \mathrm{mg} / \mathrm{mL}$. Data are reported as means + SEM $(n=18)$. ${ }^{*} p<0.02$, vs. prostaglandin $E 1$.

their shape. Figure 1 shows that paricalcitol $(100 \mathrm{nM})$ and GcMAF (1 $\mathrm{ng} / \mathrm{mL})$ completely inhibited the angiogenesis induced by PGE1.

\section{Effects of paricalcitol and GcMAF on CAMP pro- duction in human PBMCs of subjects harboring different Bsml alleles of the VDR gene}

Sixty-eight $(n=68)$ healthy donors were selected for their Bsml genotype, and 3 different groups of subjects were identified according to Bsml polymorphisms: subjects with $b b$ genotype, subjects with $B B$ genotype and subjects with $B b$ genotype.

We observed that intracellular cAMP formation in response to paricalcitol was highest in the $b b$ genotype, whereas it was not significant in the $B B$ genotype. In the $B b$ genotype, only the highest paricalcitol concentration (100 nM) elicited a statistically significant response in terms of cAMP formation (Fig. 2). GcMAF, taken as a control for its effects on angiogenesis (see also Fig. 1), significantly stimulated CAMP formation in a dose-dependent manner. Intracellular cAMP formation was highest in the $b b$ genotype, whereas it was not significant in the $B B$ genotype. In the $B b$ genotype, the response was intermediate, and the lowest concentration $(0.01 \mathrm{ng} / \mathrm{mL})$ did not elicit a statistically significant response in terms of cAMP formation. The reported increase in cAMP production following paricalcitol and GcMAF stimulation was comparable to that previously observed challenging monocytes isolated from PBMCs with 10 nM PGE2 (19).

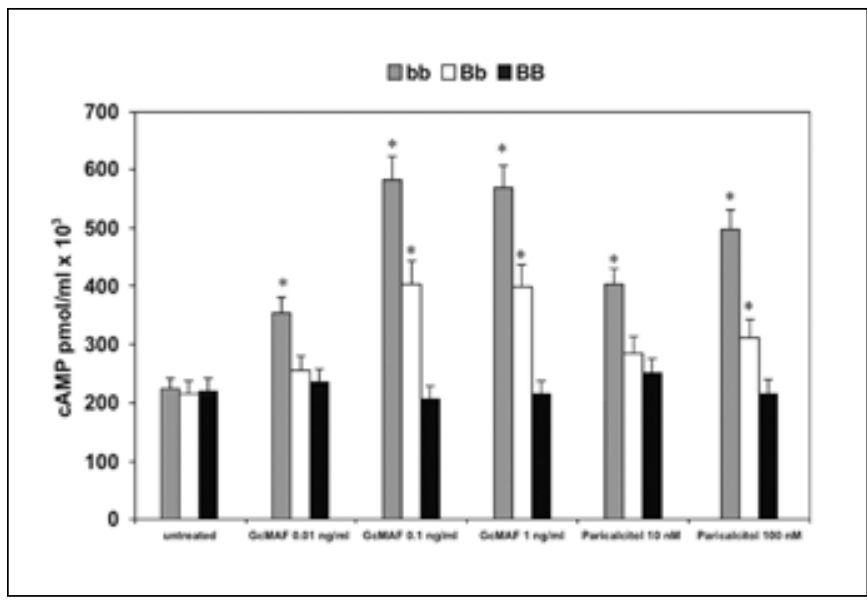

Fig. 2 - cAMP concentration in human peripheral blood mononuclear cells from subjects with different Bsml genotypes. The different compounds were added for 24 hours at the indicated concentration. Values of cAMP concentration $\left(\mathrm{pmol} / \mathrm{mL} \times 10^{3}\right)$ represent means + SEM $(n=12) .{ }^{*} p<0.02$, vs. untreated.

\section{Effects of paricalcitol and GcMAF on human PBMC viability in subjects harboring different Bsml alleles}

Vitamin $D$ inhibits macrophage function and viability by its interaction with the VDR (5); GcMAF, unlike vitamin D, is known to stimulate macrophage activation and proliferation (2). When we challenged PBMCs from subjects harboring different Bsml polymorphisms, we observed that the " $b$ " allele of the VDR gene and the bb genotype were associated with the highest inhibition of PBMC viability by paricalcitol and with the highest stimulation of proliferation by GcMAF (Fig. 3). The highest concentration of paricalcitol (100 nM) showed some (albeit not statistically significant) inhibitory effect on the $B b$ genotype. Subjects with $B b$ genotype also showed an intermediate response to GcMAF. In the homozygous $B B$ genotype, none of the concentrations of paricalcitol or GcMAF showed any significant effect. These data suggest that there is an association between the presence of " $b$ " alleles and the degree of the response to paricalcitol and GcMAF.

\section{Discussion}

Administration of vitamin $D$ is associated with greater survival in CKD patients, and increased survival appears to be independent of the effects of vitamin $D$ on mineral metabolism or parathyroid hormone levels (20). It has been hypothesized that other effects of VDR activators, defined 


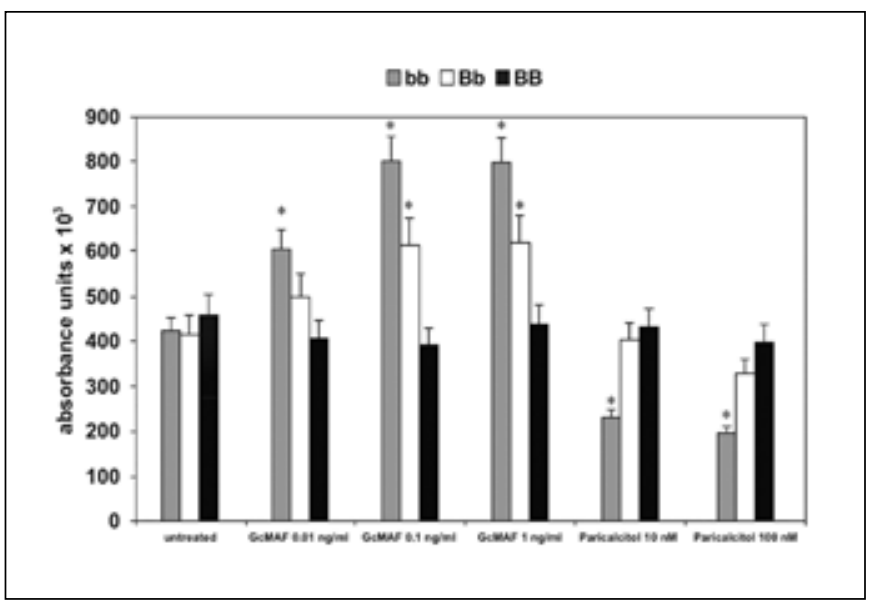

Fig. 3 - Human peripheral blood mononuclear cell viability and proliferation in subjects with different Bsml genotypes. Each compound was added to cell suspension for 24 hours at the indicated concentration. Since the background absorbance is dependent upon the culture medium, $\mathrm{pH}$, incubation time and time of exposure to light, typical background absorbance after a 1.5-hour incubation was 2-4 absorbance units $\times 10^{3}$. This value was subtracted from all reported measures. Values (absorbance units $\times 10^{3}$ ) represent means + SEM ( $n=12)$. * $p<0.02$, vs. untreated. GcMAF = Gc-macrophage activating factor.

as nonclassical, might be responsible for improved survival, because the pleiotropic actions of vitamin $D$ have been associated with improvement of cardiovascular risk factors, including increased renin activity, hypertension, inflammation, insulin resistance, diabetes and albuminuria $(21,22)$.

In this study we focused on the nonclassical effects of 2 components of the vitamin $\mathrm{D}$ axis, with particular reference to their effects on certain features of chronic inflammation that are involved in the progression of CKD - i.e., the angiogenesis stimulated by a proinflammatory prostaglandin and the activation of human PBMCs. In fact it had been demonstrated that control of angiogenesis is critical in CKD management, and the potential use of antiangiogenic peptides has been proposed as a novel therapeutic agent in diabetic nephropathy (23).

We demonstrated that paricalcitol, a nonhypercalcemic vitamin $D$ analogue already in clinical use, completely inhibited the angiogenesis induced by proinflammatory PGE1. The extent of inhibition was comparable to that obtained with GcMAF, a known inhibitor of angiogenesis pertaining to the vitamin $D$ axis but not yet in clinical use in CKD $(11,13,14)$. Thus, our results may provide the basis for exploiting the antiangiogenic properties of paricalcitol and GcMAF in CKD treatment. Our results are consistent with the reports demonstrating that inhibition of angiogenesis is associated with increased cAMP formation (24) and suggest an interconnection between vitamin $D$ and GcMAF signaling pathways. In fact, it is well established that VDR polymorphisms are associated with a variety of conditions, from cancer (25) to CKD (26), and here we demonstrate that VDR polymorphisms are also associated with the response of human PBMCs to GcMAF. These results can prove instrumental in identifying those CKD patients who would benefit the most from paricalcitol and GcMAF treatment, a treatment that is currently being proposed for other chronic conditions (8, 27-30). Assessment of the VDR genotype will also prove useful in determining the effective dosage of vitamin $D$ (or its analogues) supplementation required to reduce disease outcomes and to increase survival in CKD patients. Finally, our study demonstrates that the different components of the vitamin $D$ axis may play a role in the therapy of CKD and GCMAF, thanks to its effects on the immune system, inflammation and angiogenesis, and could soon be added to the list of compounds available for treating CKD.

Financial support: This research project has been supported by grants from the University of Firenze and by the Italian Ministry of Health (Progetto Strategico per la salute della Donna).

Conflict of interest statement: No conflict of interest exists.

Address for correspondence:

Stefania Pacini, MD

Department of Anatomy, Histology and Forensic Medicine

Viale Morgagni 85

IT-50134 Firenze, Italy

stefania.pacini@unifi.it 


\section{References}

1. Chishimba L, Thickett DR, Stockley RA, Wood AM. The vitamin $D$ axis in the lung: a key role for vitamin D-binding protein. Thorax. 2010;65(5):456-462.

2. Yamamoto N, Naraparaju VR. Immunotherapy of BALB/c mice bearing Ehrlich ascites tumor with vitamin D-binding proteinderived macrophage activating factor. Cancer Res. 1997; 57(11):2187-2192.

3. Ruggiero $M$, Pacini S. The vitamin D axis in chronic kidney disease: state of the art and future perspectives. Eur Nephrol. 2011;5:15-19.

4. Thakkinstian A, D'Este C, Eisman J, Nguyen T, Attia J. Metaanalysis of molecular association studies: vitamin $D$ receptor gene polymorphisms and BMD as a case study. J Bone Miner Res. 2004;19(3):419-428.

5. Valdivielso JM, Fernandez E. Vitamin D receptor polymorphisms and diseases. Clin Chim Acta. 2006;371(1-2):1-12.

6. Halsall JA, Osborne JE, Pringle JH, Hutchinson PE. Vitamin D receptor gene polymorphisms, particularly the novel A-1012G promoter polymorphism, are associated with vitamin D3 responsiveness and non-familial susceptibility in psoriasis. Pharmacogenet Genomics. 2005;15(5):349-355.

7. Alvarez-Hernandez D, Naves-Diaz M, Gomez-Alonso C, Coto E, Cannata-Andia JB. Tissue-specific effect of VDR gene polymorphisms on the response to calcitriol. J Nephrol. 2008; 21(6):843-849.

8. Yamamoto N, Ushijima N, Koga Y. Immunotherapy of HIVinfected patients with Gc protein-derived macrophage activating factor (GcMAF). J Med Virol. 2009;81(1):16-26.

9. Futrakul N, Butthep P, Laohareungpanya N, Chaisuriya $P$, Ratanabanangkoon K. A defective angiogenesis in chronic kidney disease. Ren Fail. 2008;30(2):215-217.

10. Chung I, Han G, Seshadri M, et al. Role of vitamin D receptor in the antiproliferative effects of calcitriol in tumor-derived endothelial cells and tumor angiogenesis in vivo. Cancer Res. 2009;69(3):967-975.

11. Pacini S, Morucci G, Punzi T, Gulisano M, Ruggiero M. Gc protein-derived macrophage-activating factor (GcMAF) stimulates cAMP formation in human mononuclear cells and inhibits angiogenesis in chick embryo chorionallantoic membrane assay. Cancer Immunol Immunother. 2010 Dec 14. [Epub ahead of print].

12. Nagasawa $H$, Uto $Y$, Sasaki $H$, et al. Gc protein (vitamin D-binding protein): Gc genotyping and GcMAF precursor activity. Anticancer Res. 2005;25(6A):3689-3695.

13. Kanda S, Mochizuki Y, Miyata Y, Kanetake H, Yamamoto N. Effects of vitamin $D(3)$-binding protein-derived macrophage activating factor (GcMAF) on angiogenesis. J Natl Cancer Inst. 2002;94(17):1311-1319.

14. Kalkunte S, Brard L, Granai CO, Swamy N. Inhibition of angiogenesis by vitamin D-binding protein: characterization of anti-endothelial activity of DBP-maf. Angiogenesis. 2005;8(4): 349-360.

15. Sardenberg C, Suassuna P, Watanabe R, et al. Balance be- tween cytokine production by peripheral blood mononuclear cells and reactive oxygen species production by monocytes in patients with chronic kidney disease. Ren Fail. 2004;26(6): 673-681.

16. Amato M, Pacini S, Aterini S, Punzi T, Gulisano M, Ruggiero M. Iron indices and vitamin $D$ receptor polymorphisms in hemodialysis patients. Adv Chronic Kidney Dis. 2008;15(2):186-190.

17. Ribatti D, Nico B, Vacca A, Presta M. The gelatin spongechorioallantoic membrane assay. Nat Protoc. 2006;1(1):85-91.

18. Giles FJ. The emerging role of angiogenesis inhibitors in hematologic malignancies. Oncology (Williston Park). 2002;16(5) (Suppl 4):23-29.

19. Takahashi HK, Liu K, Wake H, et al. Prostaglandin E2 inhibits advanced glycation end product-induced adhesion molecule expression, cytokine production, and lymphocyte proliferation in human peripheral blood mononuclear cells. J Pharmacol Exp Ther. 2009;331(2):656-670.

20. Patel TV, Singh AK. Role of vitamin D in chronic kidney disease. Semin Nephrol. 2009;29(2):113-121.

21. Cunningham J, Zehnder D. New vitamin D analogs and changing therapeutic paradigms. Kidney Int. 2011;79(7):702-707.

22. Gal-Moscovici A, Sprague SM. Use of vitamin D in chronic kidney disease patients. Kidney Int. 2010;78(2):146-151.

23. Ichinose $\mathrm{K}$, Maeshima $\mathrm{Y}$, Yamamoto $\mathrm{Y}$, et al. Antiangiogenic endostatin peptide ameliorates renal alterations in the early stage of a type 1 diabetic nephropathy model. Diabetes. 2005; 54(10):2891-2903.

24. Tsopanoglou NE, Haralabopoulos GC, Maragoudakis ME. Opposing effects on modulation of angiogenesis by protein kinase $\mathrm{C}$ and cAMP-mediated pathways. J Vasc Res. 1994;31(4):195-204.

25. Raimondi S, Johansson H, Maisonneuve P, Gandini S. Review and meta-analysis on vitamin $\mathrm{D}$ receptor polymorphisms and cancer risk. Carcinogenesis. 2009;30(7):1170-1180.

26. Tan X, He W, Liu Y. Combination therapy with paricalcitol and trandolapril reduces renal fibrosis in obstructive nephropathy. Kidney Int. 2009;76(12):1248-1257.

27. Yamamoto N, Suyama H, Nakazato H, Yamamoto N, Koga Y. Immunotherapy of metastatic colorectal cancer with vitamin D-binding protein-derived macrophage-activating factor, GcMAF. Cancer Immunol Immunother. 2008;57(7):1007-1016.

28. Yamamoto N, Suyama H, Yamamoto N, Ushijima N. Immunotherapy of metastatic breast cancer patients with vitamin D-binding protein-derived macrophage activating factor (GcMAF). Int J Cancer. 2008;122(2):461-467.

29. Yamamoto N, Suyama H, Yamamoto N. Immunotherapy for prostate cancer with Gc protein-derived macrophage activating factor, GcMAF. Transl Oncol. 2008;1(2):65-72.

30. Hayon T, Dvilansky A, Shpilberg O, Nathan I. Appraisal of the MTT-based assay as a useful tool for predicting drug chemosensitivity in leukemia. Leuk Lymphoma. 2003;44(11): 1957-1962. 